\title{
Physical activity, screen time, and sleep: do German children and adolescents meet the movement guidelines?
}

\author{
Julia Hansen $^{1} \cdot$ Reiner Hanewinkel ${ }^{1} \cdot$ Artur Galimov $^{2}$
}

Received: 8 October 2021 / Revised: 24 January 2022 / Accepted: 25 January 2022 / Published online: 3 February 2022

(c) The Author(s), under exclusive licence to Springer-Verlag GmbH Germany, part of Springer Nature 2022

\begin{abstract}
The interactions between physical activity (PA), screen time, and sleep affect the health of children and adolescents. This study described the national prevalence estimates of German youth aged 9 to 18 years who meet PA, screen time, and sleep guidelines alone and in combination and examined the associations of demographic and personal characteristics with adherence to guidelines. Data from a 2019-2020 German student survey were used $(n=15,786)$. The target population consisted of children and adolescents enrolled in grades 5-10, with a mean age of 13.0 years $(\mathrm{SD}=1.8)$ and an equal gender distribution (male: 50\%). The levels of PA, screen time, and sleep were assessed by self-reports (online questionnaires). The prevalence rates of meeting each guideline individually and in different combinations were calculated, and multilevel logistic regression models were used to examine the associations of demographic and personal characteristics with meeting versus not meeting guidelines. Overall, $9.7 \%$ of the respondents met all three guidelines combined, and approximately $25 \%$ did not meet any of the guidelines. Half of the participants (50\%) met the sleep guidelines, and approximately one third met the screen time $(35 \%)$ and PA (37\%) guidelines alone. Demographic characteristics associated with adherence to meeting all three movement guidelines included younger age, male gender, higher self-reported socioeconomic status, and school type. Personal characteristics related to adherence to meeting all three movement guidelines included better subjective school performance, less frequent attention deficit hyperactivity disorder (ADHD), lower levels of depressive symptoms, lower body mass index (BMI), and not using substances in the past 30 days.

Conclusion: A low percentage of German children and adolescents met the movement guidelines. With increasing age, the proportion of young people who follow the recommendations decreases. There is an urgent need for health interventions devoted to youth behavior as a whole.

\section{What is Known:}

- High levels of PA, low levels of screen time, and optimal sleep duration provide enhanced health benefits in comparison to the adoption of just one of these behaviors.

- Evidence shows that movement behaviors interact throughout the day and should be studied concurrently.

What is New:

- Approximately $10 \%$ of German children and adolescents met the recommendations on PA, screen time, and sleep, while $25 \%$ did not meet any guidelines.

- Meeting all guidelines was associated with less frequent ADHD and depressive symptoms, lower BMI, and less frequent substance use in the past 30 days.
\end{abstract}

Keywords Prevalence $\cdot$ Movement guidelines $\cdot$ Recommendations $\cdot$ Health behavior $\cdot$ School-aged children

Communicated by Gregorio Paolo Milani

Julia Hansen

hansen@ift-nord.de

Reiner Hanewinkel

hanewinkel@ift-nord.de

Artur Galimov

galimov@usc.edu
Institute for Therapy and Health Research, IFT-Nord, Harmsstrasse 2, 24114 Kiel, Germany

2 Department of Population and Public Health Sciences, Keck School of Medicine of University of Southern California, Institute for Health Promotion and Disease Prevention Research, Los Angeles, USA 


\section{Abbreviations}

AASM American Academy of Sleep Medicine

ADHD Attention deficit hyperactivity disorder

AOR Adjusted odds ratio

BMI Body mass index

CI Confidence interval

ED Energy drink

PA Physical activity

MICE Multivariate imputations by chained equations

SES Socioeconomic status

SDQ Strengths and Difficulties Questionnaire

WHO World Health Organization

\section{Introduction}

Modifiable lifestyle behaviors such as physical activity (PA), screen time behaviors, and sleep are considered to be relevant factors for a healthy lifestyle and affect physical health (e.g., obesity), mental and emotional health, behavioral outcomes (e.g., tobacco use), and performance-based outcomes (e.g., academic achievement) [1,2]. Getting enough uninterrupted sleep, spending low amounts of sedentary time in front of a screen, and achieving high levels of PA are each associated with better health status among youth [3-5]. The World Health Organization (WHO) recommends that children and adolescents aged 5-17 should engage in at least an average of $60 \mathrm{~min}$ per day of moderate-to-vigorous-intensity PA (primarily aerobic PA) across the week [6]. Other major US-based and UK-based PA guidelines provide similar recommendations regarding the type, intensity, volume, and duration of PA [7, 8]. In Germany, at least 90 min of daily moderate- to high-intensity exercise is recommended for young people; 60 of the overall $90 \mathrm{~min}$ can be completed through everyday activities [9]. The optimal sleep duration for 6- to 12-year-old children is 9 to $12 \mathrm{~h}$ per night; the optimal duration is 8 to $10 \mathrm{~h}$ per night for those aged 13 to 18 years [10]. Additionally, it is recommended that children and adolescents spend no more than $2 \mathrm{~h}$ per day sedentarily in front of a screen [11].

In the past, studies have commonly evaluated how time spent on one activity affects health [12]. However, the three movement-related behaviors are distributed across a $24 \mathrm{~h}$ period and fall on the movement/nonmovement intensity continuum [13]. Research has shown that the three movementrelated behaviors are codependent [14]. For optimal child health, evidence suggests that appropriate levels of PA, screen time, and sleep within $24 \mathrm{~h}$ need to be achieved simultaneously [15]. This holistic and comprehensive approach towards health behaviors is included in the Canadian $24 \mathrm{~h}$ Movement Guideline [5]. There is ongoing research aimed at determining the proportion of children and adolescents of various age groups in different countries worldwide who follow the $24 \mathrm{~h}$ movement recommendations. However, most existing studies have reported low overall adherence to the $24 \mathrm{~h}$ guideline recommendation ( 0.2 to $10 \%$ of children and adolescents) [16-24]. For Europe, research has shown that approximately 1.7 to $2.2 \%$ of European children and adolescents meet all three guidelines $[21,25,26]$.

A limited number of studies have examined the relationship between adherence to guidelines in childhood/adolescence and demographic and personal characteristics [16, 19-21, 27-29]. Among children and adolescents, favorable associations have been reported between personal characteristics - for example, better mental health indicators (e.g., less depressive symptoms), better physical health (e.g., lower body mass index (BMI)), and less risky behaviors (less substance use) - and meeting all three guidelines [1, 30, 31]. Knell et al. demonstrated that marijuana users had a lower likelihood of meeting all three recommendations compared to nonusers [1], and Tandon et al. indicated that children with attention deficit hyperactivity disorder (ADHD) are at a higher risk of not meeting the recommended amounts of PA, screen time, and sleep [32]. Some studies also suggested a positive association between academic performance and adherence to the guidelines [16, 23].

To our knowledge, there are no data on the combined movement behaviors among German children and adolescents. Previous studies conducted in Germany have focused on single behaviors. For instance, approximately $8 \%$ of girls and $16 \%$ of boys aged $14-17$ were sufficiently physically active [33], approximately $60 \%$ of 13 - to 17 -year-olds had enough sleep per night [10], and the average screen time of adolescents aged 12 to 19 years was approximately $258 \mathrm{~min}$ per day in 2020 [34]. Additionally, approximately $15 \%$ of Germans aged 3 to 17 years were overweight or obese [35]. It can therefore be assumed that a nonnegligible proportion of German children and adolescents with poor health status can be attributed to an unhealthy lifestyle. Moreover, there has been no apparent shift to a healthier lifestyle with respect to body weight or lower BMI values [36].

The literature on movement behaviors is expanding; therefore, it is critical to determine the proportions of children and adolescents who meet the PA, screen time, and sleep guidelines and better understand the characteristics associated with meeting the three movement guidelines. Hence, this study has two aims: (1) to identify the proportion of German children ( 9 to 12 years) and adolescents (13 to 18 years) meeting the PA, screen time, and sleep guidelines alone or in combination and (2) to determine the characteristics that are potentially associated with a greater risk for meeting/not meeting guidelines. These characteristics include both demographic (i.e., age, gender, school type, socioeconomic status) and personal characteristics (i.e., BMI, academic performance, 
risk-taking behavior, depression and ADHD symptoms, substance use).

\section{Methods}

\section{Participants and procedures}

Data were collected from an annual cohort study. A health survey was conducted among German students in grades $5-10$. A total of 627 schools were initially randomly selected from subregions in Germany and were invited to participate in the study. Ninety-one secondary schools from 13 federal states with 16,843 children and adolescents in grades 5-10 from 1053 classes registered in August/September 2019.

Participation in the study was voluntary. Data were collected through self-completed (paper-pencil or online version) anonymous questionnaires from October 2019 to February 2020, and thus before the start of the COVID-19 pandemic in Germany. The questionnaire consists of different sections with standardized scales or self-designed items to assess health behavior (see "Measures" section). It has been used since 2016 and is slightly modified each year, depending on the annual focus [37, 38].

Assessments were carried out in the classes during one school hour (45 min) by trained research staff or instructed school personnel. Adolescents' verbal assent was obtained on the assessment day, and parents' written informed consent was obtained 2 weeks before conducting the study. A total of 16,843 children and adolescents were included in this study.

The study was approved by the state ministries of cultural affairs, and ethical approval was obtained from the Ethical Committee of the German Psychological Society.

\section{Measures}

\section{Movement guideline adherence}

Self-reported PA was assessed using the following 4-item scale: (1)"In the past school day, on average, how much time did you spend walking?," (2)"In the past school day, on average, how much time did you spend riding a bike/kickboard/ scooter/longboard?," (3)"In the past school day, on average, how much time did you spend being physically active but were not so out of breath (i.e., light physical activities, such as inline skating with friends and playing outside)?," and (4)"In the past school day, on average, how much time did you spend being physically active and were out of breath (i.e., intense physical activities, such as jogging, participating in competitions, exercising in the gym)?." There were six response options for each statement: "not at all," "less than 10 min," "10-29 min," "30-59 min," "1-1.5 h," and "more than 1.5 h." Answers to the first three items were further averaged and served as an indicator of moderate/ daily PA, while the fourth item was an indicator of intense PA. Children and adolescents were classified as meeting PA guidelines if they participated in at least $60 \mathrm{~min}$ of moderate/ daily PA and at least $30 \mathrm{~min}$ of intense activity per day [9].

Self-reported screen time was assessed by asking, "On an average day, how much time do you usually spend in front of a screen (e.g., watching TV, watching videos, using a smartphone, browsing social networks, surfing the internet, playing video games on smartphone/computer/tablet, watching videos)?." Response options included "not at all," "less than 10 min," "up to 1 h," "1-2 h," "2-4 h," "4-8 h," and "more than 8 h." Participants who selected "not at all," "less than 10 min," "up to $1 \mathrm{~h}$," and " $1-2 \mathrm{~h}$ " (i.e., $\leq 2 \mathrm{~h}$ per day of screen time) were classified as meeting screen time guidelines [11].

The amount of sleep was assessed by the following two open-ended questions: "On an average weekday, what time do you usually go to sleep (i.e., you no longer read, no longer use a smartphone, and the light is off)?" and "On an average weekday, what time do you usually wake up?" The answers to these questions were then used to calculate the amount of sleep (in hours) that participants had on an average weekday. Furthermore, children aged 9 to 12 years were classified as meeting sleep guidelines if they reported sleeping 9 to $12 \mathrm{~h}$, while adolescents aged 13 to 18 years were classified as meeting sleep guidelines if they reported sleeping 8 to $10 \mathrm{~h} \mathrm{[10].}$

\section{Demographic and personal characteristics}

Demographic characteristics associated with health behaviors were assessed. These include age (children aged 6 to 12 years and adolescents aged 13 to 18 years), gender, socioeconomic status (SES), and school type. Selfreported SES was assessed with the MacArthur Scale [39]. Participants were asked, 'Please place an ' $\mathrm{X}$ ' on the rung that best represents where you think your family stands on the ladder?" (on a 10-point scale corresponding to a picture of a ladder, ranging from zero (low income, the worst jobs, the lowest education) to 10 (high income, the best jobs, the highest education). Regarding school type, Gymnasium (coded as 1) is the most advanced type of secondary school; other schools were coded as 0 .

The following personal characteristics were assessed: BMI, sensation seeking, school performance, current energy drink (ED) use, current substance use (cigarette smoking, e-cigarette vaping, alcohol use, hookah use), depression, and ADHD symptoms. Participants' weight 
in kilograms and height in centimeters were assessed to create the BMI variable. To address age- and sex-specific physiological changes in BMI, we created the BMI percentile variable [40]. Further participants below 5th percentile were classified as "underweight," those greater or equal 5th and below 85th percentile were classified as "healthy weight," those greater or equal 85 th and below 95th percentile were classified as "overweight," while those greater or equal 95th percentiles were classified as "obese" [41]. Risk-taking was measured by using the 2-item version of the Sensation Seeking Scale-Form V [42]: "How often do you do dangerous things to have fun?" and "How often do you do exciting things, even if they are dangerous?," each on a five-point scale ranging from "not at all" to "very often." The answers to these two items were further averaged in the risk-taking index. To assess self-reported school performance [43, 44], children and adolescents were asked, "How would you rate your school performance compared to the classmates in your class?." Response options included "much better," "somewhat better," "about the same," "somewhat worse," and "much worse." Current substance use and ED use behaviors were assessed with the following questions: "How often do you currently use cigarettes/e-cigarettes/hookah/alcohol/ energy drinks?." Response options included "not at all," "less than once a month," "at least once a month, but not every week," "at least once a week, but not every day," and "almost every day." All answers other than "not at all" and "less than once a month" were coded as past 30-day (current) use of the corresponding substance. Depression symptoms were assessed using a 5-item emotional problems subscale [45-47] of the Strengths and Difficulties Questionnaire (SDQ) (e.g., "I am often unhappy, downhearted or tearful"). The answers were further summed on the self-reported depression symptom frequency scale. Furthermore, scores from 0 to 6 were classified as "nor$\mathrm{mal} /$ borderline," while scores ranging from 7 to 10 were classified as "abnormal." Self-reported ADHD symptoms were assessed using a 5-item hyperactivity-inattention subscale [45-47] of the SDQ (e.g., "I am easily distracted, I find it difficult to concentrate.") The answers to these five items were further summed on the self-reported ADHD symptom frequency scale. Scores from 0 to 6 were classified as normal/borderline, while scores ranging from 7 to 10 were classified as abnormal.

\section{Data analysis}

Univariate analyses were conducted to assess participants' demographic and personal characteristics. Means and standard deviations (SD) were reported for continuous variables and frequencies for categorical variables. The prevalence and $95 \%$ CI of meeting none, 1, 2, or all three guidelines for the total sample and stratified by demographic and personal characteristics (for a clearer presentation, SES, school performance, and risk-taking variables were dichotomized at the median) were reported. Additionally, linear trends (i.e., $p$-trend) in meeting individual and combination of PA, screen time, and sleep guideline movement guidelines with increasing age were evaluated.

Multilevel logistic regression models with maximum likelihood estimation (ML) were used to examine the association of demographic and personal characteristics with meeting individual movement guidelines and all three guidelines simultaneously while controlling for the nesting of children and adolescents (Level 1) within 91 schools (Level 2). Separate models were constructed for each binary outcome (i.e., meeting PA guidelines, screen time guidelines, sleep time guidelines, and all three guidelines). A listwise deletion method was used to address missing data. Continuous variables (age, SES, school performance, and risk-taking variables) were assessed for normality and standardized (mean $=0, \mathrm{SD}=1$ ) to facilitate interpretation. Final data were weighted to census data to offset nonresponse bias. In parallel (sensitivity) analyses, missing data were handled using multivariate imputations by the chained equation (MICE) method for missing at random assumptions and the available covariate data; 25 imputed datasets were created. The pooling of the regression estimates followed Rubin's rule [48]. All significant differences remained and were in the same direction.

All statistical analyses were conducted using Stata software (version 15.1; Stata Corp, College Station, TX, USA). Adjusted odds ratios (AORs) with 95\% CIs were reported with statistical significance set at $p<0.05$ (2-tailed). Benjamini-Hochberg multiple testing corrections were applied to control the false discovery rate at 0.05 .

\section{Results}

After excluding participants $(n=1057)$ who had missing value data on key variables, the final analytic sample consisted of $n=15,786$ children and adolescents; half of the participants were males (50\%), and the mean age was 13.0 years $(\mathrm{SD}=1.8)$. Overall, only $9.7 \%$ [95\% CI, 9.2-10] of participants met all 3 guidelines, 25\% [95\% CI, 24-26] did not meet any of the guidelines, 39\% [95\% CI, 38-39] met at least 1 of the guidelines, and 27\% [95\% CI, 26-28] met 2 of the 3 guidelines (see Fig. 1). 
Fig. 1 Prevalence of children and adolescents meeting $0,1,2$, or 3 of the movement guidelines

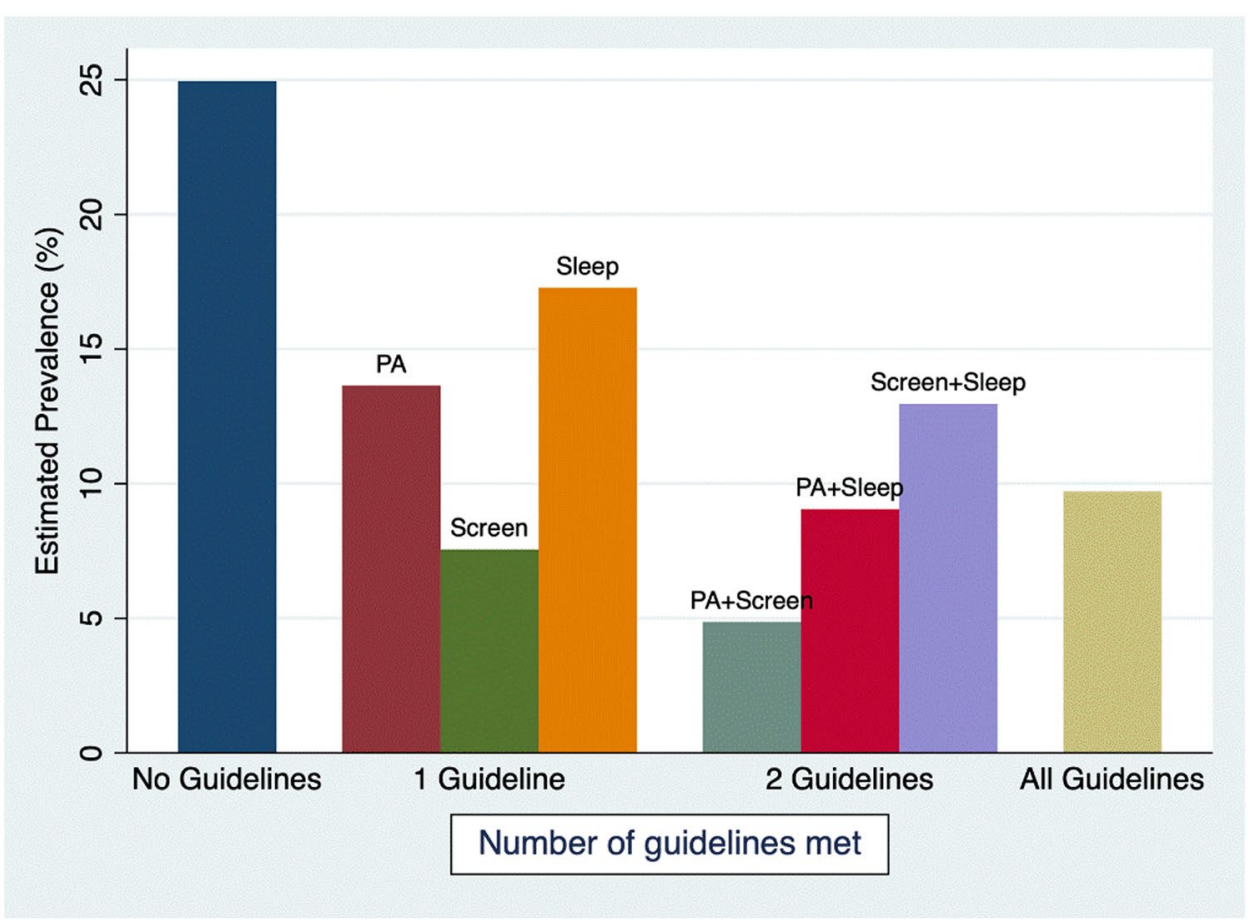

Half of the participants (50\% [95\% CI, 49-51]) met sleep guidelines, and approximately one third met screen time (35\% [95\% CI, 34-36]) and PA (37\% [95\% CI, 36-38]) guidelines (see Table 1). The estimated prevalence of meeting all three guidelines alone and combined decreased with increasing age (all $p$-trend values $<0.001$; see Fig. 2).

For instance, the prevalence of meeting all three guidelines was the highest among 9- to 10-year-old children (23\% [95\% CI, 20-25]), while only $1.7 \%$ [95\% CI, 1.1-2.6]) of 16to 18 -year-old adolescents met these guidelines $(p<0.01)$. Similarly, the prevalence of meeting sleep guidelines was the highest among 9- to 10-year-old children and 13-yearold adolescents (70\% [95\% CI, 67-73] and 60\% [95\% CI, 58-62], respectively), while it was the lowest among 16- to 18 -year-old adolescents (32\% [95\% CI, 30-35], $p<0.01$ ). Multilevel analyses revealed that boys (versus girls), those of younger age, those with lower BMI, those with higher SES, those with better school performance, those with lower selfreported ADHD and depression symptoms, and past 30-day cigarette, alcohol, and ED nonusers (versus users) were more likely to meet all three guidelines (see Table 2).

\section{Discussion}

This study examined the prevalence rates of meeting a combination of PA, screen time, and sleep guidelines among German youth $(n=15,768)$ aged 9 to 18 years. Obtaining a thorough understanding of the prevalence of meeting guidelines within $24 \mathrm{~h}$ is beneficial in promoting healthy behaviors in children and adolescents [15]. Consistent with the results of previous studies $[19,31]$, we observed that only a small proportion of German children and adolescents (approximately 10\%) spent the recommended time sleeping and physically active while limiting their screen time to $2 \mathrm{~h}$ or less within $24 \mathrm{~h}$. The prevalence of adherence to standalone PA and screen time guidelines in our sample (37\% and $35 \%$, respectively) was higher than findings of previous European studies [21, 25, 26]. Additionally, the proportion of children meeting sleep recommendations (50\%) was lower than in other studies $[19,26]$. This variation could be attributed to the differences in measurement protocol. It was demonstrated that studies utilizing self-reported measures reported a higher prevalence of meeting guidelines, while studies using objectively measured data (e.g., measurement with an accelerometer) reported a lower prevalence of meeting guidelines [17]. Hence, it seems difficult to compare the prevalence of various studies directly, as the measurement protocol varies across studies (particularly concerning PA measurement). There is a clear need for high-quality studies that use robust and validated measures of all movement behaviors [16].

The second finding of this study concerns the characteristics associated with adherence to PA, screen time, and sleep recommendations. It was found that the majority of the characteristics in the multiple models were independently associated with guideline adherence. In terms of gender, findings on differences between boys and girls in the present study are in line with previous studies, showing that boys are more likely to comply with PA guidelines 
Table 1 Prevalence of German children and adolescents meeting movement guidelines (PA/screen time/sleep) weighted column \%

\begin{tabular}{|c|c|c|c|c|c|c|}
\hline & Unweighted total sample & None & PA & Screen time & Sleep & PA + screen time + sleep \\
\hline Overall & $15,786(100 \%)$ & $25(24-26)$ & $37(36-38)$ & $35(34-36)$ & $50(49-51)$ & $9.7(9.2-10)$ \\
\hline \multicolumn{7}{|l|}{ Demographic characteristics } \\
\hline \multicolumn{7}{|l|}{ Gender $(n=15,580)^{\mathrm{a}}$} \\
\hline Male & 7807 & $22(21-23)$ & $41(40-42)$ & $35(34-36)$ & $51(50-52)$ & $11(9.9-11)$ \\
\hline Female & 7773 & $28(26-29)$ & $32(31-33)$ & $35(34-36)$ & $49(48-50)$ & $9.0(8.3-9.7)$ \\
\hline \multicolumn{7}{|l|}{ Age $(n=15,786)^{\mathrm{a}}$} \\
\hline $9-12$ years & 6451 & $16(15-17)$ & $40(38-41)$ & $55(54-56)$ & $57(56-58)$ & $16(15-17)$ \\
\hline $13-18$ years & 9335 & $32(30-33)$ & $35(34-36)$ & $21(20-22)$ & $45(44-46)$ & $5.3(4.8-5.8)$ \\
\hline \multicolumn{7}{|l|}{ School type $(n=15,786)^{\mathrm{a}}$} \\
\hline Gymnasium & 9566 & $22(21-23)$ & $42(41-43)$ & $41(40-42)$ & $51(50-52)$ & $13(13-14)$ \\
\hline Other & 6220 & $27(26-28)$ & $33(32-34)$ & $30(29-31)$ & $49(47-50)$ & $7.1(6.4-7.7)$ \\
\hline \multicolumn{7}{|l|}{ Self-report SES $(n=15,695)^{\mathrm{a}}$} \\
\hline Below median $(<7)$ & 6256 & $28(27-29)$ & $33(32-34)$ & $31(30-33)$ & $48(47-50)$ & $7.6(7.0-8.3)$ \\
\hline Above median $(\geq 7)$ & 9439 & $23(22-24)$ & $40(39-41)$ & $37(36-38)$ & $51(50-52)$ & $11(11-12)$ \\
\hline \multicolumn{7}{|l|}{ Personal characteristics } \\
\hline \multicolumn{7}{|l|}{ BMI percentile $(n=15,353)^{\mathrm{a}}$} \\
\hline Underweight & 910 & $20(17-23)$ & $37(33-40)$ & $45(42-49)$ & $55(51-58)$ & $13(11-16)$ \\
\hline Normal & 12,454 & $24(23-25)$ & $37(37-38)$ & $36(35-37)$ & $51(50-52)$ & $10(9.8-11)$ \\
\hline Overweight & 1562 & $32(30-35)$ & $34(31-36)$ & $24(22-27)$ & $41(39-44)$ & $5.8(4.7-7.1)$ \\
\hline Obese & 427 & $32(28-37)$ & $29(25-34)$ & $22(18-26)$ & $48(42-53)$ & $4.3(2.6-7)$ \\
\hline \multicolumn{7}{|c|}{ School performance $(n=15,764)^{\mathrm{a}}$} \\
\hline Below median $(<3)$ & 3429 & $31(30-33)$ & $31(30-33)$ & $25(24-27)$ & $43(41-45)$ & $4.6(3.9-5.4)$ \\
\hline Above median $(\geq 3)$ & 12,335 & $23(22-24)$ & $38(37-39)$ & $38(37-38)$ & $52(51-53)$ & $11(11-12)$ \\
\hline \multicolumn{7}{|l|}{ Risk-taking $(n=15,684)^{\mathrm{a}}$} \\
\hline Low $(<2)$ & 5721 & $21(20-22)$ & $32(31-34)$ & $43(41-44)$ & $59(58-61)$ & $12(12-13)$ \\
\hline $\operatorname{High}(\geq 2)$ & 9963 & $27(26-28)$ & $39(38-40)$ & $30(29-31)$ & $45(44-46)$ & $8.2(7.6-8.7)$ \\
\hline \multicolumn{7}{|l|}{ Depression scale $(n=15,728)^{\mathrm{a}}$} \\
\hline Normal/borderline (0-6) & 14,113 & $23(23-24)$ & $37(37-38)$ & $36(35-37)$ & $52(51-52)$ & $10(9.8-11)$ \\
\hline Abnormal (7-10) & 1615 & $40(37-42)$ & $30(28-33)$ & $20(18-23)$ & $34(32-37)$ & $3.8(2.9-4.9)$ \\
\hline \multicolumn{7}{|l|}{ ADHD scale $(n=15,701)^{\mathrm{a}}$} \\
\hline Normal/borderline (0-6) & 14,426 & $24(23-25)$ & $37(36-38)$ & $36(35-36)$ & $51(50-52)$ & $10(9.6-11)$ \\
\hline Abnormal (7-10) & 1275 & $34(31-37)$ & $34(31-37)$ & $26(23-28)$ & $37(34-40)$ & $5.2(4.0-6.5)$ \\
\hline \multicolumn{7}{|l|}{ Past 30-day substance user } \\
\hline Cigarettes $(n=15,758)^{\mathrm{a}}$ & 823 & $45(42-49)$ & $30(27-33)$ & $14(12-17)$ & $27(24-30)$ & $1.0(0.5-1.9)$ \\
\hline E-cigarettes $(n=15,743)^{\mathrm{a}}$ & 444 & $39(34-44)$ & $41(36-46)$ & $14(10-17)$ & $28(24-33)$ & $2.2(1.2-4.1)$ \\
\hline Hookah $(n=15,746)^{\mathrm{a}}$ & 519 & $38(34-43)$ & $38(34-43)$ & $13(11-17)$ & $28(24-32)$ & $10.0(9.6-10.5)$ \\
\hline Alcohol $(n=15,758)^{\mathrm{a}}$ & 2163 & $41(39-43)$ & $37(35-39)$ & $13(11-14)$ & $29(27-31)$ & $1.9(1.4-2.6)$ \\
\hline Energy drinks $(n=15,763)^{\mathrm{a}}$ & 2666 & $39(37-41)$ & $34(32-36)$ & $14(12-15)$ & $33(31-35)$ & $2.3(1.8-3.0)$ \\
\hline
\end{tabular}

$A O R$ adjusted odds ratio; $C I$ confidence interval; $P A$ physical activity; $S E S$ socioeconomic status; $B M I$ body mass index; $A D H D$ attention deficit hyperactivity disorder

${ }^{a}$ Number of subjects with complete (non-missing) information on this variable

[19]. Academic performance was associated with adherence to all guidelines, regardless of whether the former was measured by attending a specific school type or the selfassessment of how good one's performance is compared to that of classmates. These findings are consistent with the results of a 2017 Canadian study on the associations of adherence to recommendations and academic performance among adolescents [16]. One of the explanations for the observed associations is that the adoption of a healthy lifestyle has been shown to be one of the most important correlates of brain development processes and cognitive function [49]. Furthermore, studies have shown PA to have positive effects on cognition, while media use, particularly excessive television viewing and video game playing, has 
Fig. 2 Prevalence of meeting the movement guidelines by age

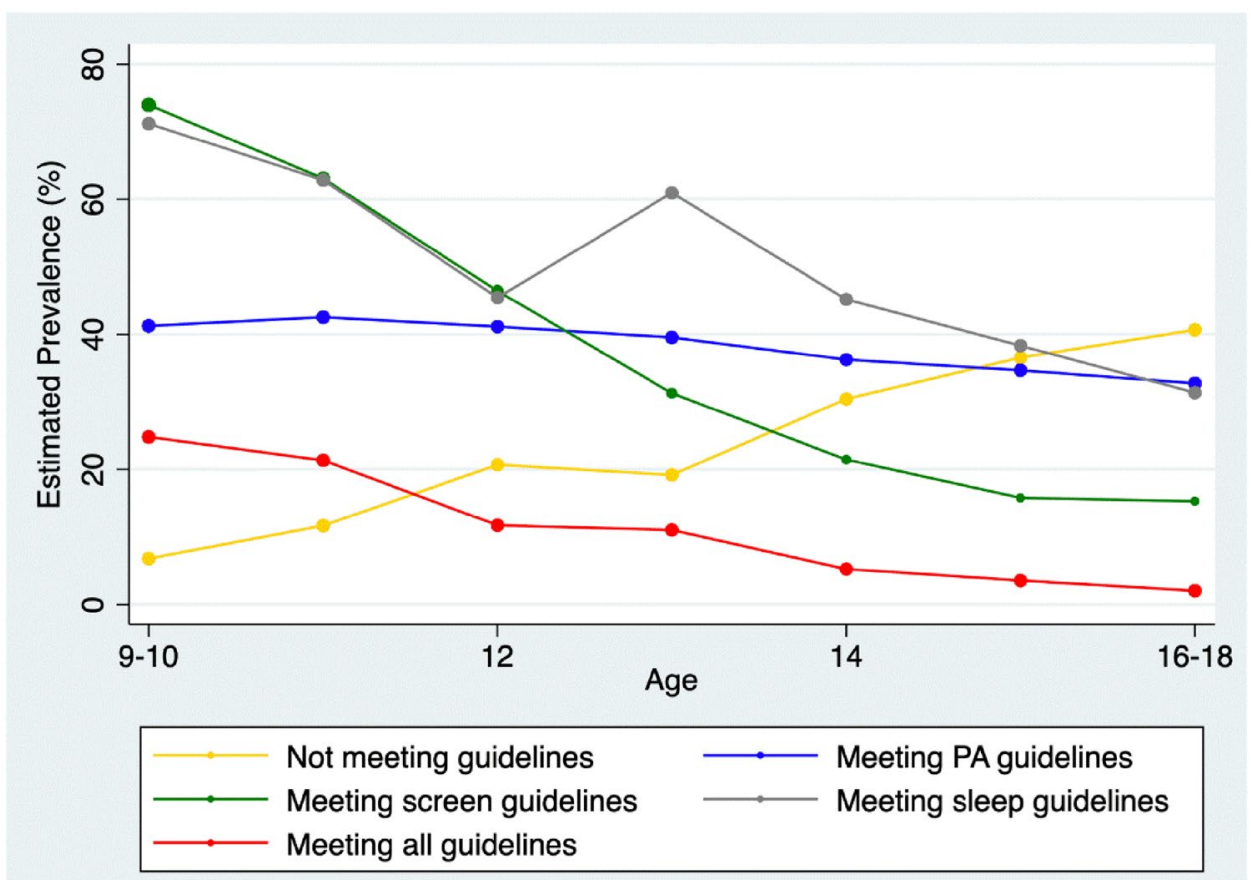

Table 2 Adjusted relative odds of German children and adolescents meeting movement guidelines (PA/screen time/sleep)

\begin{tabular}{|c|c|c|c|c|}
\hline & $\begin{array}{l}\text { PA } \\
\text { AOR (95\% CI) }\end{array}$ & $\begin{array}{l}\text { Screen time } \\
\text { AOR }(95 \% \mathrm{CI})\end{array}$ & $\begin{array}{l}\text { Sleep } \\
\text { AOR }(95 \% \text { CI })\end{array}$ & $\begin{array}{l}\text { PA + screen time + sleep } \\
\text { AOR }(95 \% \text { CI })\end{array}$ \\
\hline \multicolumn{5}{|l|}{ Demographic characteristics } \\
\hline Gender $($ female $=$ reference group $)$ & $1.31(1.20-1.43)^{*}$ & $0.97(0.88-1.06)$ & $1.10(1.01-1.20)^{*}$ & $1.24(1.09-1.40)^{*}$ \\
\hline $\mathrm{Age}^{\mathrm{a}}$ & $0.90(0.87-0.94) *$ & $0.44(0.41-0.48)^{*}$ & $0.75(0.71-0.80)^{*}$ & $0.52(0.48-0.57)^{*}$ \\
\hline School type (other $=$ reference group) & $1.41(1.29-1.55)^{*}$ & $1.43(1.18-1.72)^{*}$ & $0.93(0.83-1.06)$ & $1.62(1.41-1.86)^{*}$ \\
\hline Self-report SES ${ }^{a}$ & $1.10(1.06-1.13)^{*}$ & $1.06(1.01-1.12)^{*}$ & $1.06(1.02-1.10)^{*}$ & $1.15(1.08-1.22) *$ \\
\hline \multicolumn{5}{|l|}{ Personal characteristics } \\
\hline BMI percentile $^{\mathrm{a}}$ & $0.94(0.91-0.97)^{*}$ & $0.84(0.80-0.88)^{*}$ & $0.92(0.89-0.96)^{*}$ & $0.84(0.80-0.89)^{*}$ \\
\hline School performance ${ }^{a}$ & $1.13(1.08-1.18)^{*}$ & $1.06(0.99-1.13)$ & $1.01(0.96-1.05)$ & $1.16(1.09-1.24) *$ \\
\hline Risk-taking $^{\mathrm{a}}$ & $1.27(1.22-1.33)^{*}$ & $0.91(0.87-0.95)^{*}$ & $0.82(0.78-0.85)^{*}$ & $0.94(0.88-1.01)$ \\
\hline $\begin{array}{l}\text { Depression scale }(\text { normal/borderline }=\text { reference } \\
\text { group) }\end{array}$ & $0.94(0.90-0.99)^{*}$ & $0.77(0.72-0.81)^{*}$ & $0.81(0.77-0.86)^{*}$ & $0.79(0.73-0.86)^{*}$ \\
\hline ADHD scale (normal/borderline $=$ reference group $)$ & $0.91(0.87-0.96)^{*}$ & $0.82(0.77-0.87)^{*}$ & $0.90(0.87-0.94)^{*}$ & $0.83(0.78-0.89) *$ \\
\hline \multicolumn{5}{|l|}{ Past 30-day substance use (no use $=$ reference group) } \\
\hline Cigarettes & $0.67(0.55-0.82)^{*}$ & $1.51(1.10-2.06)^{*}$ & $0.94(0.78-1.14)$ & $0.35(0.14-0.87) *$ \\
\hline E-cigarettes & $1.29(1.01-1.66)$ & $0.87(0.58-1.32)$ & $0.97(0.70-1.34)$ & $1.15(0.56-2.37)$ \\
\hline Hookah & $1.07(0.88-1.30)$ & $1.10(0.82-1.49)$ & $0.90(0.69-1.16)$ & $1.14(0.55-2.36)$ \\
\hline Alcohol & $1.10(0.97-1.23)$ & $0.71(0.58-0.87)^{*}$ & $0.70(0.62-0.79)^{*}$ & $0.56(0.40-0.80)^{*}$ \\
\hline Energy drinks & $0.91(0.80-1.03)$ & $0.47(0.41-0.55)^{*}$ & $0.65(0.58-0.73) *$ & $0.46(0.34-0.62)^{*}$ \\
\hline
\end{tabular}

$A O R$ adjusted odds ratio; $C I$ confidence interval; $P A$ physical activity; SES socioeconomic status; $B M I$ body mass index; $A D H D$ attention deficit hyperactivity disorder

Significant results $p<0.05$ displayed in bold

*Statistically significant after Benjamini-Hochberg corrections for multiple testing to control false-discovery rate at 0.05 (based on 2-tailed corrected $p$ value)

${ }^{a}$ Rescaled (mean $=0, \mathrm{SD}=1$ ) such that the ORs indicate the change in odds in the outcome associated with an increase in $1 \mathrm{SD}$ unit on the continuous covariate scale 
been shown to decrease attention and cognitive functioning, which may impair academic outcomes [50-52].

Personal characteristics associated with adherence to the guidelines also include ADHD and depressive symptoms. Vulnerable subgroups such as children and adolescents with abnormal scores on the depression scale were less likely to comply with all three movement guidelines, which is consistent with cross-sectional studies from the USA and the UK $[1,53,54]$. Evidence suggests that PA is inversely associated with depressive symptoms $[55,56]$ and may also be beneficial for curbing depression [57]; thus, PAtargeted interventions may be promising. Patte et al. examined whether changes in adherence to PA, screen time, and sleep guidelines were associated with depression symptoms among adolescents and concluded that complying with sleep recommendations emerged as the most consistent predictor of depression symptoms [58]. From this perspective, one could hypothesize that promoting healthy sleep habits could play an important role in preventing or even reducing youth depressive symptoms. Children and adolescents with abnormal scores on the ADHD scale were also less likely to spend the optimal amount of time being physically active, in front of a screen or sleeping. Hence, children and adolescents who had these conditions may be at a higher risk of not meeting the guidelines and need to be targeted via movement behavior interventions promoting PA, less screen time, and proper sleep.

Substance use is associated with adverse health outcomes $[59,60]$. We observed that past 30-day cigarette, alcohol, and ED use were associated with a lower level of compliance with all three movement guidelines, while past 30-day e-cigarette and hookah use were not related with adherence. Hence, substance users were at greater risk for nonadherence to recommended lifestyle behaviors. One can speculate that nonusers, compared to users, could have better selfregulation, which goes along with living a healthier lifestyle, including more PA, limited screen time, and better sleep habits. There is evidence that adherence to the movement behavior guidelines is associated with better social health, such as better self-regulation, decision-making, and psychosocial functioning [61].

A higher BMI that carries the risk of developing overweight or obesity and increases the risk of developing chronic diseases was associated with not meeting any guidelines. Previous work has found similar relationships with BMI and compliance with health behavior guidelines that underscore the need for interventions [22].

The third relevant result of this study supports previous work on the age effect. From ages 9 to 18 years, the prevalence of meeting all and each specific guideline decreased, with less than $5 \%$ of 16- to 18-year-olds meeting all guidelines simultaneously compared to more than $20 \%$ of 9 to 10 -year-olds adhering to all guidelines. The sharpest decrease is observed for meeting screen time recommendations, which was also found in a US sample [31]. These results are particularly alarming given the growing evidence suggesting that movement behaviors in adolescence are predictive of movement behaviors in adulthood [62].

Although this study provides information about characteristics associated with meeting or not meeting the guidelines, many questions remain, especially concerning the temporality and causal relationships between combinations of physical activity, screen time, and sleep duration and health indicators and the underlying mechanisms explaining how and why certain combinations of behaviors may be beneficial. Hence, there is a strong need for prospective, high-quality studies to increase the understanding in this topic area [30]. Thus far, we also cannot estimate the effects of the COVID-19 pandemic on compliance with the movement guidelines. To examine these effects on children's and adolescents' movement behaviors, the presented data provide information about the level of compliance before the outbreak of COVID-19 and could serve as reference values for German adolescents.

\section{Limitations}

The study has several strengths, including a demographically diverse and large sample and statistical control for important covariates. Nonetheless, several limitations should be considered. The inference of causality between adherence to the movement guidelines and outcomes is precluded because of the cross-sectional design. Hence, we cannot imply a causeeffect relationship with our study. In addition, the data were collected through self-report. Considering school performance, children and adolescents seem to be quite good at assessing their self-performance, as self-reported grades were found to be highly positively correlated with actual grades. On the other hand, it is known that biases in the self-reporting of PA levels cannot be ruled out. Likewise, self-reports of screen time might be biased, and using a single item to assess the screen time may lead to reliability issues. Future research should assess the association with objectively measured PA to improve the reliability of results (e.g., using accelerometers). Although accelerometers can estimate the $24 \mathrm{~h}$ movement behavior more precisely, selfreported measurements are an ideal measurement method for large-scale monitoring and surveillance due to their lower testing effort and lower costs. The present study did not measure specific types of PA or types of screen time activities (gaming vs. social media). There is evidence that different types of PA and different types of screen time activities could be differentially associated with mental health problems and substance use in children and adolescents [63, 64]. Furthermore, sleep time was assessed only on weekdays, 
which may have affected the study findings, as for children of this age group, there may be differences in sleep duration between weekdays and weekend days. Finally, the health survey was limited to children and adolescents attending schools interested in a health promotion project, which suggests a health-promoting school environment that generally places more emphasis on healthy lifestyles.

\section{Conclusion}

Only $10 \%$ of German children and adolescents are sufficiently physically active, spend the optimal time, in front of a screen, and sleep. Adherence to PA and screen time guidelines were lower than meeting sleep guidelines and were consequently the primary contributing factors for not meeting all three movement behaviors in combination. With increasing age, fewer adolescents adhere to recommended PA, screen, and sleep time levels, and more adolescents do not meet any of the three guidelines. Therefore, there is an urgent need to develop health promotion programs that target a healthy $24 \mathrm{~h}$ period, including an optimal distribution of time spent with PA, in front of a screen, and sleeping.

Acknowledgements We would like to thank all schools, teachers, and students for their collaboration.

Authors' contributions Julia Hansen and Reiner Hanewinkel contributed to the study conception and design. Material preparation, data collection, and analysis were performed by Julia Hansen, Reiner Hanewinkel, and Artur Galimov. The first draft of the manuscript was written by Julia Hansen and Artur Galimov and all authors commented on previous versions of the manuscript. All authors read and approved the final manuscript.

Funding This study was funded by DAK-Gesundheit.

Data availability Data available on request from the corresponding authors.

\section{Declarations}

Ethics approval Ethical approval was obtained from the Ethical Committee of the German Psychological Society (Reference number: RH 042015_1), and the study implementation was approved by the ministries of cultural affairs of the involved Federal states.

Consent to participate Adolescents' verbal assent was obtained on the assessment day and parents' written informed consent were obtained two weeks before conducting the study.

Consent for publication Written informed consent of the parents was collected.

Conflict of interest The authors declare no competing interests.

\section{References}

1. Knell G et al (2019) Prevalence and likelihood of meeting sleep, physical activity, and screen-time guidelines among US youth. JAMA Pediatr 173(4):387-389

2. Saunders TJ et al (2016) Combinations of physical activity, sedentary behaviour and sleep: relationships with health indicators in school-aged children and youth. Appl Physiol Nutr Metab 41(6 Suppl 3):S283-S293

3. Poitras VJ et al (2016) Systematic review of the relationships between objectively measured physical activity and health indicators in school-aged children and youth. Appl Physiol Nutr Metab 41(6 Suppl 3):S197-239

4. Carson V et al (2016) Systematic review of sedentary behaviour and health indicators in school-aged children and youth: an update. Appl Physiol Nutr Metab 41(6 Suppl 3):S240-S265

5. Tremblay MS et al (2016) Canadian 24-hour movement guidelines for children and youth: an integration of physical activity, sedentary behaviour, and sleep. Applied Physiology, Nutrition, and Metabolism 41(6 (Suppl. 3)):S311-S327

6. WHO (2020) WHO guidelines on physical activity and sedentary behaviour. W.H. Organization, Editor. World Health Organization: Geneva

7. Piercy KL et al (2018) The physical activity guidelines for Americans. JAMA 320(19):2020-2028

8. Care D.o.H.a.S., (2019) Physical activity guidelines: UK Chief Medical Officers' Report. Department of Health and Social Care: London, UK

9. Pfeifer K et al (2016) Nationale Empfehlungen für Bewegung und Bewegungsförderung. [cited 2021 8.1.]. Available from: https://www.bunde sgesundheitsministerium.de/fileadmin/Dateien/3_Downloads/B/ Bewegung/Nationale-Empfehlungen-fuer-Bewegung-und-Beweg ungsfoerderung-2016.pdf

10. Paruthi $\mathrm{S}$ et al (2016) Recommended amount of sleep for pediatric populations: a consensus statement of the American Academy of Sleep Medicine. J Clin Sleep Med 12(6):785-786

11. Communications Co. and Media, Children, adolescents, and the media (2013) Pediatrics 132(5):958-961

12. Rosenberger ME et al (2019) The 24-hour activity cycle: a new paradigm for physical activity. Med Sci Sports Exerc 51(3):454-464

13. Carson V, Tremblay MS, Chastin SFM (2017) Cross-sectional associations between sleep duration, sedentary time, physical activity, and adiposity indicators among Canadian preschoolaged children using compositional analyses. BMC Public Health 17(Suppl 5):848

14. Chaput JP, Saunders TJ, Carson V (2017) Interactions between sleep, movement and other non-movement behaviours in the pathogenesis of childhood obesity. Obes Rev 18(Suppl 1):7-14

15. Rollo S, Antsygina O, Tremblay MS (2020) The whole day matters: understanding 24-hour movement guideline adherence and relationships with health indicators across the lifespan. J Sport Health Sci 9(6):493-510

16. Lien A et al (2020) Adherence to 24-hour movement guidelines and academic performance in adolescents. Public Health 183:8-14

17. Chen ST et al (2020) Meeting 24-hour movement guidelines: prevalence, correlates and the relationships with overweight and obesity among Chinese children and adolescents. J Sport Health Sci

18. da Costa, B.G.G., et al., Prevalence and sociodemographic factors associated with meeting the 24-hour movement guidelines in a sample of Brazilian adolescents. PLoS One, 2020. 15(9): p. $\mathrm{e} 0239833$.

19. Tapia-Serrano MA, Sevil-Serrano J, Sánchez-Miguel PA (2021) Adherence to 24-hour movement guidelines among Spanish adolescents: differences between boys and girls. Children (Basel) 8(2) 
20. De Craemer M et al (2018) Compliance with 24-h movement behaviour guidelines among Belgian pre-school children: the ToyBox-Study. Int J Environ Res Public Health 15(10)

21. Rubín L et al (2020) Prevalence and correlates of adherence to the combined movement guidelines among Czech children and adolescents. BMC Public Health 20(1):1692

22. Roman-Viñas B et al (2016) Proportion of children meeting recommendations for 24-hour movement guidelines and associations with adiposity in a 12-country study. Int J Behav Nutr Phys Act 13(1):123

23. Howie EK et al (2020) Associations between meeting sleep, physical activity or screen time behaviour guidelines and academic performance in Australian school children. BMC Public Health 20(1):520

24. Bang F et al (2020) Physical activity, screen time and sleep duration: combined associations with psychosocial health among Canadian children and youth. Health Rep 31(5):9-16

25. Jakubec $\mathrm{L}$ et al (2020) Is adherence to the 24-hour movement guidelines associated with a reduced risk of adiposity among children and adolescents? BMC Public Health 20(1):1119

26. Sevil-Serrano J et al (2019) Healthy or unhealthy? The cocktail of health-related behavior profiles in Spanish adolescents. Int J Environ Res Public Health 16(17)

27. Lee EY et al (2019) Levels and correlates of 24-hour movement behaviors among South Koreans: results from the Korea National Health and Nutrition Examination Surveys, 2014 and 2015. J Sport Health Sci 8(4):376-385

28. Chen B et al (2019) Socio-demographic and maternal predictors of adherence to 24-hour movement guidelines in Singaporean children. Int J Behav Nutr Phys Act 16(1):70

29. Manyanga T et al (2019) Prevalence and correlates of adherence to movement guidelines among urban and rural children in Mozambique: a cross-sectional study. Int J Behav Nutr Phys Act 16(1):94

30. Sampasa-Kanyinga $\mathrm{H}$ et al (2020) Combinations of physical activity, sedentary time, and sleep duration and their associations with depressive symptoms and other mental health problems in children and adolescents: a systematic review. Int J Behav Nutr Phys Act 17(1):72

31. Friel CP et al (2020) U.S. children meeting physical activity, screen time, and sleep guidelines. Am J Prev Med 59(4):513-521

32. Tandon PS et al (2019) Physical activity, screen time, and sleep in children with ADHD. J Phys Act Health 16(6):416-422

33. Finger JD et al (2018) Körperliche Aktivität von Kindern und Jugendlichen in Deutschland - Querschnittergebnisse aus KiGGS Welle 2 und Trends. Journal of Health Monitoring 3(1)

34. Feierabend SRT, Kheredmand, H, Glöckler S (2020) JIM-Studie 2020 - Jugend, Information, Medien. Basisuntersuchung zum Medienumgang 12- bis 19-Jähriger in Deutschland

35. Schienkiewitz A (2018) Übergewicht und Adipositas im Kindesund Jugendalter in Deutschland - Querschnittergebnisse aus KiGGS Welle 2 und Trends.

36. Schienkiewitz A et al (2019) Body mass index among children and adolescents: prevalences and distribution considering underweight and extreme obesity : Results of KiGGS Wave 2 and trends. Bundesgesundheitsblatt Gesundheitsforschung Gesundheitsschutz 62(10): $1225-1234$

37. Galimov A et al (2019) Energy drink consumption among German adolescents: prevalence, correlates, and predictors of initiation. Appetite 139:172-179

38. Hansen J, Hanewinkel R, Morgenstern M (2018) Electronic cigarette marketing and smoking behaviour in adolescence: a cross-sectional study. ERJ open research 4(4):00155-02018

39. Goodman E et al (2001) Adolescents' perceptions of social status: development and evaluation of a new indicator. J Pediatrics 108(2):e31-e31
40. Kromeyer-Hauschild K et al (2001) Perzentile für den Bodymass-Index für das Kindes- und Jugendalter unter Heranziehung verschiedener deutscher Stichproben. Percentiles of body mass index in children and adolescents evaluated from different regional German studies [in German]. Monatsschrift Kinderheilkunde 149(8):807-818

41. Weir CB, Jan A (2019) BMI classification percentile and cut off points. Stat Pearls [Internet]

42. Stephenson MT et al (2003) Brief measures of sensation seeking for screening and large-scale surveys. Drug Alcohol Depend 72(3):279-286

43. Achenbach TM (1991) Manual for the youth self-report and 1991 profile. Department of Psychiatry University of Vermont Burlington, VT.

44. Sharif I, Sargent JD (2006) Association between television, movie, and video game exposure and school performance. Pediatrics 118(4):e1061-e1070

45. Goodman R (2001) Psychometric properties of the Strengths and Difficulties Questionnaire. J Am Acad Child Adolesc Psychiatry 40(11):1337-1345

46. Goodman R et al (2003) Using the Strengths and Difficulties Questionnaire (SDQ) to screen for child psychiatric disorders in a community sample. Int Rev Psychiatry 15(1-2):166-172

47. Muris P, Meesters C, van den Berg F (2003) The Strengths and Difficulties Questionnaire (SDQ). Eur Child Adolesc Psychiatry 12(1):1-8

48. White IR, Royston P, Wood AM (2011) Multiple imputation using chained equations: issues and guidance for practice. Stat Med 30(4):377-399

49. Naveed S, Lakka T, Haapala EA (2020) An overview on the associations between health behaviors and brain health in children and adolescents with special reference to diet quality. Int J Environ Res Public Health 17(3):953

50. Donnelly JE et al (2016) Physical activity, fitness, cognitive function, and academic achievement in children: a systematic review. Med Sci Sports Exerc 48(6):1197-1222

51. Nathanson AI et al (2014) The relation between television exposure and executive function among preschoolers. Dev Psychol 50(5):1497-1506

52. Adelantado-Renau M et al (2019) Association between screen media use and academic performance among children and adolescents: a systematic review and meta-analysis. JAMA Pediatr 173(11):1058-1067

53. Zhu X, Haegele JA, Healy S (2019) Movement and mental health: behavioral correlates of anxiety and depression among children of 6-17 years old in the U.S. Mental Health and Physical Activity 16:60-65

54. Pearson N, Sherar LB, Hamer M (2019) Prevalence and correlates of meeting sleep, screen-time, and physical activity guidelines among adolescents in the United Kingdom. JAMA Pediatr 173(10): $1-2$

55. Gordon BR et al (2018) Association of efficacy of resistance exercise training with depressive symptoms: meta-analysis and meta-regression analysis of randomized clinical trials. JAMA Psychiat 75(6):566-576

56. Eime RM et al (2013) A systematic review of the psychological and social benefits of participation in sport for children and adolescents: informing development of a conceptual model of health through sport. Int J Behav Nutr Phys Act 10:98

57. Biddle SJH, Asare M (2011) Physical activity and mental health in children and adolescents: a review of reviews. Br J Sports Med 45(11):886-895

58. Patte KA et al (2020) Are one-year changes in adherence to the 24-hour movement guidelines associated with depressive symptoms among youth? BMC Public Health 20(1):793

59. Hall WD et al (2016) Why young people's substance use matters for global health. Lancet Psychiatry 3(3):265-279 
60. Degenhardt $\mathrm{L}$ et al (2016) The increasing global health priority of substance use in young people. Lancet Psychiatry 3(3):251-264

61. Janssen I, Roberts KC, Thompson W (2017) Is adherence to the Canadian 24-Hour Movement Behaviour Guidelines for Children and Youth associated with improved indicators of physical, mental, and social health? Appl Physiol Nutr Metab 42(7):725-731

62. Lounassalo I et al (2019) Distinct trajectories of physical activity and related factors during the life course in the general population: a systematic review. BMC Public Health 19(1):271
63. Brailovskaia J, Velten J, Margaf J (2019) Relationship between daily stress, depression symptoms, and facebook addiction disorder in Germany and in the United States. Cyberpsychol Behav Soc Netw 22(9):610-614

64. Wang D et al (2014) Impact of physical exercise on substance use disorders: a meta-analysis. PLoS One 9(10):e110728

Publisher's Note Springer Nature remains neutral with regard to jurisdictional claims in published maps and institutional affiliations. 